\title{
STANDARDISATION AND COMPARISON OF DIFFERENT METHODS FOR PREPARING PLATELET RICH PLASMA
}

Lakshita Varshney1, Seema Gupta²

1Resident, Department of Immunohematology and Blood Transfusion, MGM Medical College and Hospital, Kamothe, Navi Mumbai. ${ }^{2}$ Associate Professor, Department of Immunohematology and Blood Transfusion, MGM Medical College and Hospital, Kamothe, Navi Mumbai.

\begin{tabular}{l}
\hline ABSTRACT \\
BACKGROUND \\
Platelet rich plasma (PRP) is defined as a volume of the plasma fraction of autologous blood with an above baseline platelet \\
concentration. Platelet releases the growth factors from their granules that are beneficial for tissue healing. Therefore, platelet rich \\
plasma is being increasingly used in various medical and surgical fields. As such, there are no defined guidelines available for PRP \\
preparation. Hence, this study is aimed to develop a protocol for an optimised method of PRP preparation by standardisation of two \\
different methods, namely tube method and syringe method in our setup.
\end{tabular}

\section{MATERIALS AND METHODS}

The prospective comparative study was conducted at MGM Blood Bank, Kamothe, Navi Mumbai, for a period of 3 months. Blood samples were collected from 10 voluntary healthy donors. PRP was prepared by double centrifugation method. Four protocols of PRP preparation were defined for both tube and syringe method each. Platelet count and platelet increment were analysed and tabulated.

\section{RESULTS}

The reliable, practical and cost-effective protocol for platelet rich plasma preparation in our setup was found to be $1200 \mathrm{rpm}$ for 12 mins in first spin and 2000 rpm for 10 mins in second spin for tube method and $900 \mathrm{rpm}$ for 8 mins in first spin and 2500 rpm for 10 mins in second spin for syringe method in our setup.

\section{CONCLUSION}

With some limitations, our study determined the protocols for PRP preparation by two simple and cost-effective techniques. Syringe method was found more effective in terms of platelet increment and sterility in our setup. Therefore, the study may contribute towards future researches where a defined protocol of PRP preparation and clinical benefits are still needed to be explored.

\section{KEYWORDS}

Platelet Rich Plasma, Tube Method, Syringe Method.

HOW TO CITE THIS ARTICLE: Varshney L, Gupta S. Standardisation and comparison of different methods for preparing platelet rich plasma. J. Evolution Med. Dent. Sci. 2017;6(90):6325-6329, DOI: 10.14260/jemds/2017/1376

\section{BACKGROUND}

Platelet rich plasma (PRP) is defined as a volume of the plasma fraction of autologous blood with an above baseline platelet concentration. ${ }^{1}$ Although, the optimal PRP platelet concentration is unclear, the current methods by which PRP is prepared report 3- to 5 -fold increase over the baseline.2,3 Platelet concentrations of $200 \times 10^{3}$ platelets/ $\mu \mathrm{L}$ up to $1000 \mathrm{x}$ $10^{3}$ platelets $/ \mu \mathrm{L}$ are considered therapeutic for tissue healing. 4

Platelets play a significant role in haemostasis by preventing blood loss through platelet plug formation. Platelets release the growth factors from their granules such as platelet-derived GF (PDGF), transforming GF- $\beta 1$ (TGF- $\beta 1$ ), vascular endothelial GF (VEGF), basic fibroblastic GF (bFGF), insulin like growth factor-1 (IGF-1), hepatocyte growth factor

'Financial or Other Competing Interest': None.

Submission 13-10-2017, Peer Review 05-11-2017,

Acceptance 11-11-2017, Published 20-11-2017.

Corresponding Author:

Dr. Lakshita Varshney,

C-2/119A, Lawrence Road,

Keshav Puram, Delhi-110035.

E-mail: lakshita1989v@gmail.com

DOI: $10.14260 /$ jemds/2017/1376
(HGF) and epidermal GF (EGF) that promote beneficial effects on tissue healing and influence the reactivity involved in angiogenesis and inflammation. 4,5

At the beginning of the 21st century, the clinical application of platelet-rich plasma (PRP) was considered a breakthrough in the stimulation and acceleration of bone and soft tissue healing. ${ }^{6}$ Since then, it is increasingly used in almost all fields of surgery and medicine like Plastic Surgery, Orthopaedic Surgery, Cardiac Surgery, Ophthalmology, and Sports Medicine.7,8 PRP is obtained by treating blood samples with various methods such as single centrifugation, double centrifugation or apheresis. Also, it must be calcium activated before being applied over the surgical site. ${ }^{9,10}$

As such there are no guidelines available for standardisation of PRP preparation and use. Many protocols have been tried by various authors previously. Different methods of preparation may result in different platelet concentrations and each preparation may have different applications. For this reason, the development of autologous formulations with a controlled platelet counting appears to be challenging. ${ }^{11}$ The present study is proposed to develop a protocol for an optimised method of PRP preparation by standardisation of two different methods, namely tube method and syringe method. This will focus on desired 
platelet yield in the produced product and will also contribute to future studies.

\section{MATERIALS AND METHODS}

The prospective comparative study was conducted in the Department of Immunohematology and Blood Transfusion, MGM Medical College and Hospital, Kamothe, Navi Mumbai for a period of 3 months from January 2017 to March 2017. The study was approved by the Institutional Ethical Committee.

\section{Blood Collection}

Blood samples were obtained from 10 healthy adult volunteers at MGM Hospital Blood Bank, Kamothe, Navi Mumbai. The volunteers comprised of 7 males and 3 females ranging in age from 20 - 45 years (Mean age 31.4 years). Informed consent was obtained from each volunteer before collecting the samples.

$161 \mathrm{~mL}$ of whole blood was withdrawn from each healthy volunteer using a butterfly needle. The whole blood to anticoagulant ratio was maintained as 1: 9. The tubes were let to stand for a minimum of half an hour. Out of $161 \mathrm{~mL}, 80 \mathrm{~mL}$ of whole blood was used for tube method, $80 \mathrm{~mL}$ for syringe method and $1 \mathrm{~mL}$ was taken in blood collection tube (BD Vacutainer).

\section{Platelet Rich Plasma Preparation}

Platelet rich plasma (PRP) is prepared by differential centrifugation, wherein acceleration force is adjusted to sediment certain cellular constituents based on different specific gravity. PRP can be prepared by the PRP method or by the buffy-coat method. In our study, PRP double centrifugation method was used. In this method, an initial centrifugation to separate red blood cells (RBC) is followed by a second centrifugation to concentrate platelets, which are suspended in the smallest final plasma volume. ${ }^{12}$ Flow chart of platelet rich plasma preparation by double centrifugation method is described in Figure 1.

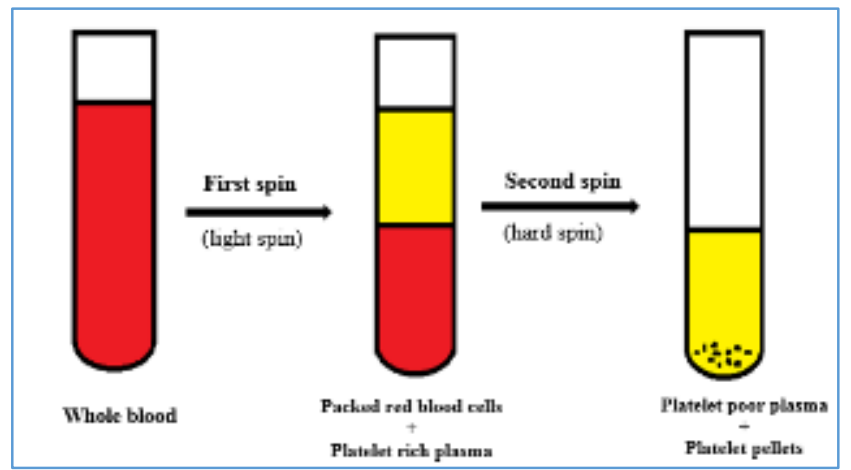

Figure 1. Double Centrifugation Process of PRP Preparation

For tube method, four $20 \mathrm{~mL}$ borosilicate glass tubes containing $20 \mathrm{~mL}$ whole blood were prepared covered with sterile caps for first spin. Another four $20 \mathrm{~mL}$ glass tubes were prepared for second spin. Four variations of centrifugations were used for platelet rich plasma preparation-

- A: $1100 \mathrm{rpm}$ for $12 \mathrm{mins}$ in first spin and $2100 \mathrm{rpm}$ for 10 mins in second spin.
- B: $1200 \mathrm{rpm}$ for 12 mins in first spin and $2000 \mathrm{rpm}$ for 10 mins in second spin.

- $\quad$ C: $1400 \mathrm{rpm}$ for 12 mins in first spin and $2000 \mathrm{rpm}$ for 10 mins in second spin.

- D: $1500 \mathrm{rpm}$ for 12 mins in first spin and $2000 \mathrm{rpm}$ for 10 mins in second spin.

The tubes were placed in a refrigerated centrifuge (Cryofuge Heraeus 5500i) and were subjected to first light spin at $22^{\circ} \mathrm{C}$. The whole blood on centrifugation got separated into red blood cells, white blood cells and platelet rich plasma. PRP was drawn off from the appropriate level and again centrifuged (second heavy spin) to separate the platelets at $22^{\circ} \mathrm{C}$. Supernatant plasma was discarded and around $5 \mathrm{~mL}$ was left along with the platelet pellet. This PRP was transferred to a sterile tube and kept in platelet agitator for an hour [Figure 2 (i) and 2(ii)].

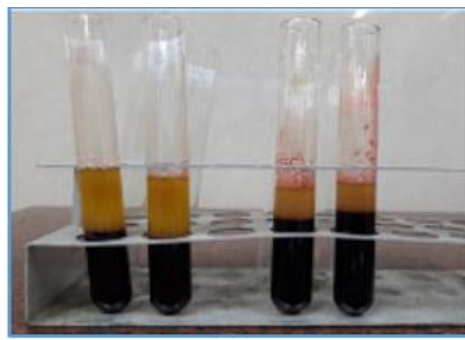

(I)

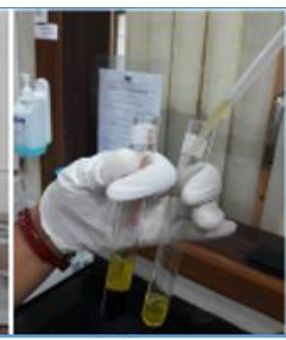

(II)
Figure 2. PRP Preparation by Tube Method

Similarly, for syringe method, eight $50 \mathrm{~mL}$ sterile syringes were prepared with sterile caps for first and second spin having $20 \mathrm{~mL}$ blood in it. Another four variations of centrifugations were used for platelet rich plasma preparation-

- a: $1200 \mathrm{rpm}$ for 12 mins in first spin and $2200 \mathrm{rpm}$ for 10 mins in second spin.

- b: $1200 \mathrm{rpm}$ for $10 \mathrm{mins}$ in first spin and $2500 \mathrm{rpm}$ for 10 mins in second spin.

- c: $1100 \mathrm{rpm}$ for $8 \mathrm{mins}$ in first spin and $2500 \mathrm{rpm}$ for 10 mins in second spin.

- d: $900 \mathrm{rpm}$ for 8 mins in first spin and $2500 \mathrm{rpm}$ for 10 mins in second spin.

The syringes were kept in buckets of refrigerated centrifuge (Cryofuge Heraeus 5500i) and were subjected to first light spin at $22^{\circ} \mathrm{C}$. PRP was then transferred to another sterile syringe using a sterile connecting tube. This was again centrifuged (second heavy spin) to separate the platelets at $22^{\circ} \mathrm{C}$. The supernatant plasma was discarded and around 5 $\mathrm{mL}$ is left along with the concentrated platelet. This PRP was then transferred to a sterile tube and kept in platelet agitator for an hour [Figure 3(i) and 3(ii)]

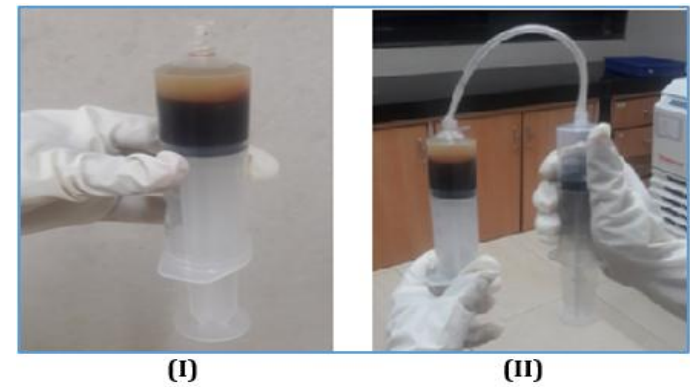

Figure 3. PRP Preparation by Syringe Method 


\section{Platelet Count}

Platelet counts were obtained using 5-part haematological analyser (Celltac $\alpha$, Nihon Kohden India Pvt. Ltd.) from whole blood samples and platelet rich plasma. In addition to platelet counts, one important parameter was also evaluated for the PRP samples, i.e. platelet increment (folds). Platelet increment was calculated using the following equation: ${ }^{9}$

Platelet Increment $=\frac{\text { Platelet count of PRP- Platelet count of whole blood }}{}$ Platelet count of whole blood

\section{Statistical Methods}

The data was entered, tabulated and evaluated using Microsoft Excel and SPSS Software ver. 20. The mean platelet count and mean platelet increment (folds) were calculated for both syringe and tube methods. The results are mentioned as Mean \pm Standard Deviation. The significance of differences between the platelet counts obtained by four protocols of tube method as well as syringe method were determined by ANOVA (Analysis of Variance) test. The significance of differences between the platelet counts of whole blood and PRP was determined by paired ' $\mathrm{t}$ ' test. $\mathrm{P}$ value of $<0.05$ was considered significant. The correlation between platelet counts and platelet increment was established by Pearson's ' $r$ ' correlation coefficient, where the value range was +1 to -1 .

\section{RESULTS}

During the 3-month study period, 10 whole blood samples were analysed for platelet rich plasma preparation by tube and syringe technique. The samples were collected from healthy adult volunteers. Among the 10 volunteers, 7 were males and 3 were females with age ranging from 20 - 45 years (Mean age $31.4 \pm 7.91$ years).

The baseline whole blood platelet count ranged from 170 $\mathrm{x} 10^{3} / \mu \mathrm{L}$ to $403 \times 10^{3} / \mu \mathrm{L}$ with mean platelet count of $256.4 \pm$ $82.10 \times 10^{3} / \mu \mathrm{L}$.

The values of platelet count and platelet increment (fold) at different centrifugations by tube method are summarised in Table 1 and 2. It showed that mean platelet counts by four protocols of tube method were A $\left(998.50 \pm 350.38 \times 10^{3} / \mu \mathrm{L}\right.$ at $1100 \mathrm{rpm}$ for $12 \mathrm{mins}$ in first spin and $2100 \mathrm{rpm}$ for 10 mins in second spin), B (1478.84 $\pm 424.83 \times 10^{3} / \mu \mathrm{L}$ at 1200 rpm for 12 mins in first spin and $2000 \mathrm{rpm}$ for $10 \mathrm{mins}$ in second spin $), C\left(1338.51 \pm 382.11 \times 10^{3} / \mu \mathrm{L}\right.$ at $1400 \mathrm{rpm}$ for 12 mins in first spin and $2000 \mathrm{rpm}$ for $10 \mathrm{mins}$ in second spin) and $D\left(1067.80 \pm 351.61 \times 10^{3} / \mu \mathrm{L}\right.$ at $1500 \mathrm{rpm}$ for 12 mins in first spin and $2000 \mathrm{rpm}$ for $10 \mathrm{mins}$ ) in second spin. The mean platelet increment was $2.86 \pm 0.28$ fold, $4.83 \pm 0.34$ fold, $4.41 \pm 0.27$ fold and $2.89 \pm 0.28$ fold respectively.

\begin{tabular}{|l|c|c|c|c|c|}
\hline \multicolumn{2}{|c|}{} & \multicolumn{3}{|c|}{$\begin{array}{c}\text { Platelet Count after Centrifugation } \\
(\mathbf{1 0 3}) / \mu \mathrm{L}\end{array}$} \\
\hline & $\begin{array}{c}\text { Baseline } \\
\text { Platelet } \\
\text { Count }(\mathrm{x} \\
\left.10^{3}\right) / \mu \mathrm{L}\end{array}$ & $\mathrm{A}$ & $\mathrm{B}$ & $\mathrm{C}$ & $\mathrm{D}$ \\
& & & & \\
\hline Sample 1 & 203 & 653.66 & 1220.03 & 1057.63 & 789.67 \\
\hline Sample 2 & 170 & 654.5 & 1050.6 & 952 & 736.1 \\
\hline Sample 3 & 243 & 967.14 & 1397.25 & 1278.18 & 901.53 \\
\hline Sample 4 & 141 & 509.01 & 829.08 & 781.14 & 589.38 \\
\hline Sample 5 & 403 & 1624.09 & 2285.01 & 1970.67 & 1692.6 \\
\hline
\end{tabular}

\begin{tabular}{|c|c|c|c|c|c|}
\hline Sample 6 & 314 & 1164.94 & 1657.92 & 1591.98 & 1199.48 \\
\hline Sample 7 & 285 & 1128.6 & 1727.1 & 1422.15 & 1174.2 \\
\hline Sample 8 & 342 & 1368 & 1788.66 & 1751.04 & 1497.96 \\
\hline Sample 9 & 195 & 797.55 & 1195.35 & 1039.35 & 869.7 \\
\hline $\begin{array}{c}\text { Sample } \\
10\end{array}$ & 268 & 1117.56 & 1637.48 & 1541 & 1227.44 \\
\hline $\begin{array}{c}\text { Mean } \\
\text { Platelet } \\
\text { Count }\end{array}$ & 256.40 & 998.50 & 1478.84 & 1338.51 & 1067.80 \\
\hline $\begin{array}{c}\text { Standard } \\
\text { Deviation }\end{array}$ & 82.10 & 350.38 & 424.83 & 382.11 & 351.61 \\
\hline $\begin{array}{c}\text { A: } 1100 \text { rpm for 12 mins in first spin and } 2100 \text { rpm for } 10 \\
\text { mins in second spin }\end{array}$ \\
B: 1200 rpm for 12 mins in first spin and 2000 rpm for 10 \\
mins in second spin \\
C: 1400 rpm for 12 mins in first spin and 2000 rpm for 10 \\
mins in second spin \\
D: 1500 rpm for 12 mins in first spin and 2000 rpm for 10 \\
mins in second spin \\
\hline \multicolumn{7}{|c|}{ Table 1. Platelet Count with Different Centrifugations by Method } \\
\hline
\end{tabular}

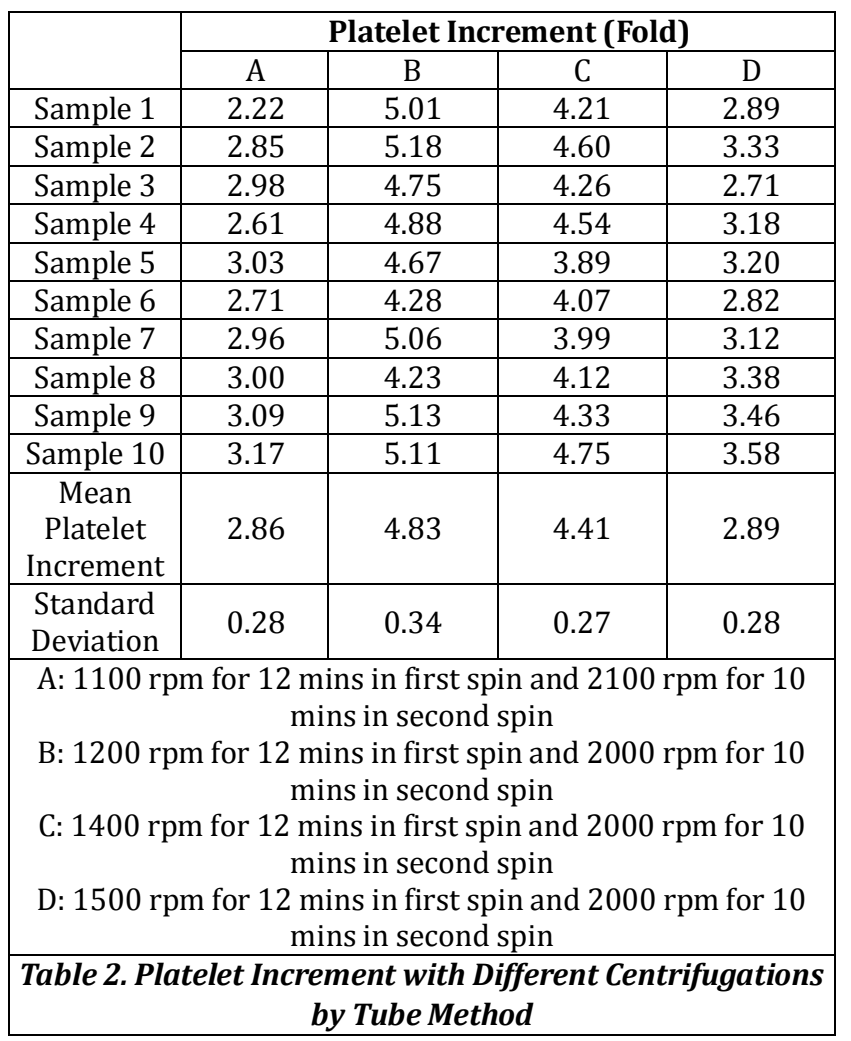

Similarly, the values of platelet count and platelet increment (fold) at different centrifugations by syringe method are summarised in Table 3 and 4 . It showed that mean platelet counts by four protocols of syringe method were 'a' $\left(979.57 \pm 376.54 \times 10^{3} / \mu \mathrm{L}\right.$ at $1200 \mathrm{rpm}$ for $12 \mathrm{mins}$ in first spin and $2200 \mathrm{rpm}$ for 10 mins in second spin), 'b' $\left(782.71 \pm 270.11 \times 10^{3} / \mu \mathrm{L}\right.$ at $1200 \mathrm{rpm}$ for $10 \mathrm{mins}$ in first spin and $2500 \mathrm{rpm}$ for 10 mins in second spin), ' $c$ ' (1177.23 \pm $409.75 \times 10^{3} / \mu \mathrm{L}$ at $1100 \mathrm{rpm}$ for $8 \mathrm{mins}$ in first spin and 2500 rpm for 10 mins in second spin) and 'd' (1518.30 $\pm 508.71 \mathrm{x}$ $10^{3} / \mu \mathrm{L}$ at $900 \mathrm{rpm}$ for 8 mins in first spin and $2500 \mathrm{rpm}$ for 10 mins in second spin). The mean platelet increment was found to be $2.79 \pm 0.40$ fold, $2.02 \pm 0.36$ fold, $3.57 \pm 0.35$ fold and $4.88 \pm 0.31$ fold respectively. 


\begin{tabular}{|c|c|c|c|c|c|}
\hline & \multicolumn{4}{|c|}{$\begin{array}{l}\text { Platelet Count after Centrifugation } \\
\qquad\left({\left.\mathrm{x} 10^{3}\right) / \mu \mathrm{L}}^{-}\right.\end{array}$} \\
\hline & $\begin{array}{c}\text { Baseline } \\
\text { Platelet } \\
\text { Count } \\
\left(\mathrm{x} 10^{3}\right) / \mu \mathrm{L}\end{array}$ & A & B & $\mathrm{C}$ & D \\
\hline Sample 1 & & 779.52 & 609 & 933.8 & 1187.55 \\
\hline Sample 2 & & & 6 & 839.8 & \\
\hline & & & & & \\
\hline & & & & 592 & \\
\hline Sam & & 185 & 25 & 2006.94 & 377.7 \\
\hline & & & & 1372 & 9.94 \\
\hline Sam & & & & .5 & \\
\hline Sam & & 121 & & 1545.84 & 2.82 \\
\hline Sam & & & & 963.3 & 1072.5 \\
\hline $\begin{array}{r}\text { Samp } \\
10 \\
\end{array}$ & 268 & 956.76 & 766.48 & 1240.84 & 1661.6 \\
\hline $\begin{array}{c}\text { Mean } \\
\text { Platelet } \\
\text { Count }\end{array}$ & 256.40 & 979.57 & 782.71 & 1177.23 & 1518.30 \\
\hline $\begin{array}{l}\text { Standard } \\
\text { Deviation }\end{array}$ & 82.10 & 376.54 & 270.11 & 409.75 & 508.71 \\
\hline \multicolumn{6}{|c|}{ 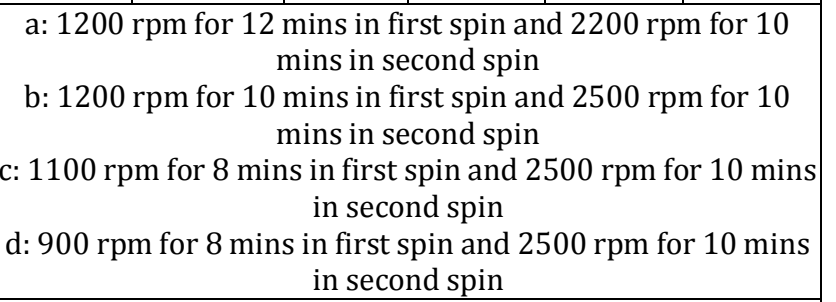 } \\
\hline \multicolumn{6}{|c|}{$\begin{array}{l}\text { Table 3. Platelet Count with Different Centrifugations by } \\
\text { Syringe Method }\end{array}$} \\
\hline
\end{tabular}

\begin{tabular}{|c|c|c|c|c|}
\hline & \multicolumn{4}{|c|}{ Platelet Increment (fold) } \\
\cline { 2 - 5 } & $\mathrm{A}$ & $\mathrm{B}$ & $\mathrm{C}$ & $\mathrm{D}$ \\
\hline Sample 1 & 2.84 & 2.00 & 3.60 & 4.85 \\
\hline Sample 2 & 3.19 & 1.98 & 3.94 & 5.09 \\
\hline Sample 3 & 2.08 & 2.31 & 2.86 & 5.06 \\
\hline Sample 4 & 2.68 & 1.20 & 3.20 & 4.25 \\
\hline Sample 5 & 3.60 & 1.75 & 3.98 & 4.90 \\
\hline Sample 6 & 2.70 & 2.45 & 3.37 & 5.21 \\
\hline Sample 7 & 2.76 & 2.04 & 3.70 & 5.08 \\
\hline Sample 8 & 2.54 & 2.25 & 3.52 & 4.71 \\
\hline Sample 9 & 3.01 & 2.39 & 3.94 & 4.50 \\
\hline Sample 10 & 2.57 & 1.86 & 3.63 & 5.20 \\
\hline $\begin{array}{c}\text { Mean } \\
\text { Platelet } \\
\text { Increment }\end{array}$ & 2.79 & 2.02 & 3.57 & 4.88 \\
\hline $\begin{array}{c}\text { Standard } \\
\text { Deviation }\end{array}$ & 0.40 & 0.36 & 0.35 & 0.31 \\
\hline
\end{tabular}

a: $1200 \mathrm{rpm}$ for 12 mins in first spin and $2200 \mathrm{rpm}$ for 10 mins in second spin

b: $1200 \mathrm{rpm}$ for $10 \mathrm{mins}$ in first spin and $2500 \mathrm{rpm}$ for 10 mins in second spin

c: $1100 \mathrm{rpm}$ for $8 \mathrm{mins}$ in first spin and $2500 \mathrm{rpm}$ for $10 \mathrm{mins}$ in second spin

d: $900 \mathrm{rpm}$ for $8 \mathrm{mins}$ in first spin and $2500 \mathrm{rpm}$ for $10 \mathrm{mins}$ in second spin

Table 4. Platelet Increment with Different Centrifugations by Syringe Method

In tube method, the statistical differences between the protocols A, B, C and D were significant $(\mathrm{p}<0.05)$ by ANOVA test, hence protocol 'B' (1200 rpm for 12 mins in first spin and $2000 \mathrm{rpm}$ for 10 mins in second spin) was chosen as standardised protocol. Similarly, with Syringe method, protocols a, b, c and d showed significant statistical differences $(\mathrm{p}<0.05)$ by ANOVA test, hence protocol ' $\mathrm{d}$ ' $(900$ rpm for 8 mins in first spin and $2500 \mathrm{rpm}$ for $10 \mathrm{mins}$ in second spin) was chosen as standardised protocol. The platelet counts of whole blood and PRP obtained by tube method as well as the syringe method showed a significant statistical difference $(\mathrm{p}<0.05)$ using paired ' $\mathrm{t}$ ' test. The platelet counts and platelet increment obtained by the chosen protocols of both tube and syringe technique showed no statistical difference on comparison ( $p>0.05)$ by ANOVA test. A positive correlation (value, $r=1$ ) by Pearson's correlation coefficient was seen between the platelet counts and platelet increment obtained by both the methods.

Hence, the reliable and practical platelet rich plasma preparation protocol was found to be $1200 \mathrm{rpm}$ for $12 \mathrm{mins}$ in first spin and $2000 \mathrm{rpm}$ for 10 mins in second spin for tube method and $900 \mathrm{rpm}$ for 8 mins in first spin and $2500 \mathrm{rpm}$ for 10 mins in second spin for syringe method in our setup.

\section{DISCUSSION}

Platelet rich plasma (PRP) is an autologous product, which contains many growth factors. These factors are required for wound healing and tissue repair. There are very few studies to determine the clinical benefits of platelet rich plasma. Also, there are only few studies to ascertain the preparation methods of PRP and their standardisation. However, understanding the differences in PRP preparations is essential when interpreting the clinical study results. Currently, different clinical settings have defined their own protocol for PRP preparation and no standard protocol of PRP preparation has been established as yet. Hence, many studies are required to be done to investigate the efficacy of standardised preparations of Platelet Rich Plasma (PRP).13

Platelet rich plasma can be prepared by single centrifugation protocol or double centrifugation protocol. ${ }^{3}$ Like various other studies, our study is based on double centrifugation protocol to identify maximum platelet enrichment upon PRP preparation.

The three major variables that affect the recovery of cells from the whole blood by double centrifugation protocol are rotor size, speed and duration of centrifugation. These are important in determining the quality and clinical effectiveness of platelet concentrate preparations. The rotor size is constant for a given centrifuge, but if the radius becomes altered by any method the centrifugal force must be calculated by real radius. Hence, we standardised our protocols by altering the other two variables, i.e. the speed and the duration of centrifugation. ${ }^{3,14,15}$ Using the hit and trial method, the speed and duration of centrifugation were formulated and the protocols were defined according to our centrifugation conditions. The speed was kept lower, as higher speed may affect the platelet functionality.

In our study, we prepared PRP by tube method and syringe method. In both of these methods, four different protocols of varying speed and duration of centrifugation were used. The first centrifugation was kept at lowest speed and shortest time, as it was aimed to separate red blood cells from other component of whole blood. Thereafter, the second centrifugation was kept at high speed and longer, as it was aimed to separate platelets from plasma. With higher speed and longer time, more platelets will be precipitated.14,16

We found that the mean platelet increment was $4.83 \pm$ 0.34 for tube method when centrifuged at $1200 \mathrm{rpm}$ for 12 mins in first spin and 2000 rpm for 10 mins in second spin. 
For syringe method, we found that the mean platelet increment was $4.88 \pm 0.31$ at $900 \mathrm{rpm}$ for 8 mins in first spin and $2500 \mathrm{rpm}$ for $10 \mathrm{mins}$ in second spin in our setup. Our results were found to be comparable with the results of other studies. Pourmokhtar et al concluded that $2100 \times \mathrm{g}$ for 2.30 mins in the first centrifugation step and $4150 \times g$ for 6 mins in the second centrifugation step yielded the greatest platelet recovery and the highest enrichment capacity. ${ }^{3}$ Mazzocca et al analysed three protocols for preparing PRP samples with different compositions: a low platelet $\left(382 \times 10^{3} / \mathrm{mm}^{3}\right)$ and low WBC $\left(0.6 \times 10^{3} / \mathrm{mm}^{3}\right)$ process with one spin step at 1500 rpm for 5 mins (10 mL WB); a high platelet $\left(940 \times 10^{3} / \mathrm{mm}^{3}\right)$ and high WBC $\left(17 \times 10^{3} / \mathrm{mm}^{3}\right)$ process with one spin step at $3200 \mathrm{rpm}$ for $15 \mathrm{mins}$ ( $27 \mathrm{~mL} \mathrm{WB}$ ) and a double-spin process (1500 rpm for 5 mins and $6300 \mathrm{rpm}$ for $20 \mathrm{mins}$ ) that produced a higher platelet concentration $\left(472 \times 10^{3} / \mathrm{mm}^{3}\right)$ and lower WBC $\left(1.5 \times 103 / \mathrm{mm}^{3}\right) .{ }^{4}$ In another study by Bausset et al, the mean platelet concentration factor of 3.47 was found on two serial 15 mins centrifugations of anticoagulated whole blood at 130 and $250 \times$ g successively, which was optimal for platelet functionality and growth factor release capacity. ${ }^{11}$ Similarly, Dhurat et al found that at $900 \mathrm{~g} \times 5 \mathrm{mins}$ for 1 st centrifugation and $1000 \mathrm{~g} \times 10 \mathrm{mins}$ for 2nd centrifugation at $16^{\circ} \mathrm{C}$ in a refrigerated centrifuge, the PRP yield was found to be optimum. The authors have consistently recorded platelet count exceeding 10 lakhs $/ \mathrm{mL}$ using these parameters. ${ }^{12}$ Perez et al observed that the efficient conditions for platelet recovery are low centrifugal acceleration (close to $100 \times \mathrm{g}, 10$ minutes) in the first spin and around $400 \times \mathrm{g}$ in the second spin for preventing effects on activating platelets. ${ }^{17}$

Some limitations to the study were also observed. The borosilicate tubes needed to be autoclaved every time for use. If not sealed properly, the sterility of the product would be breeched. This could overcome by use of sterile and disposable syringes. Also baseline platelet value varies from person to person, which leads to difficulty in suggesting the fixed concentrations and clinical benefits. ${ }^{7}$ In all the techniques used for PRP preparation in our study are cost effective and can be implemented according to the set up since not much statistical variation was found in final standardised protocols obtained by both the methods. However, syringe method is still preferable due to its sterile condition being a closed system. These techniques further require minimal training of the staff and utilise minimum time for preparation.

\section{CONCLUSION}

Our study determined the protocols for PRP preparation by two simple and cost-effective techniques. We found that out of the two procedures, PRP preparation by syringe method was more effective in terms of platelet increment and sterility in our setup. This study may be useful for various settings where commercial kits cannot be used due to higher cost. Also, it may be beneficial for future researches in this field as a defined protocol of PRP preparation and clinical benefits are still needed to be explored.

\section{REFERENCES}

[1] Gkini MA, Kouskoukis AE, Tripsianis G, et al. Study of platelet-rich plasma injections in the treatment of androgenetic alopecia through an one-year period.
Journal of Cutaneous and Aesthetic Surgery 2014;7(4):213-9.

[2] Khatu SS, More YE, Gokhale NR, et al. Platelet-rich plasma in androgenic alopecia: myth or an effective tool. Journal of Cutaneous and Aesthetic Surgery 2014;7(2):107-10.

[3] Pourmokhtar M, Moghaddam SE, Abbasi F, et al. Comparative study of four platelet-rich plasma methods for preparing platelet concentrates. IJBC 2014;6(3):1037.

[4] Mazzocca AD, McCarthy MB, Chowaniec DM, et al. Platelet-rich plasma differs according to preparation method and human variability. J Bone Joint Surg Am 2012;94(4):308-16.

[5] Cole BJ, Seroyer ST, Filardo G, et al. Platelet-rich plasma: where are we now and where are we going? Sports Health 2010;2(3):203-10.

[6] Lippross, Sebastian, Alini M. Platelet-rich plasma for bone healing-to use or not to use? AO Dialogue 2007;01:25-9.

[7] Kececi Y, Ozsu S, Bilgir 0. A cost-effective method for obtaining standard platelet-rich plasma. Wounds 2014;26(8):232-8.

[8] Marlovits S, Mousavi M, Gäbler C, et al. A new simplified technique for producing platelet-rich plasma: a short technical note. Eur Spine J 2004;13(Suppl 1):S102-S6.

[9] Tamimi FM, Montalvo S, Tresguerres I, et al. A comparative study of 2 methods for obtaining plateletrich plasma. J Oral Maxillofac Surg 2007;65(6):1084-93.

[10] Stover EF. Intraoperatively prepared platelet gel as an alternative to fibrin glue in dural wound repair. Transfusion 1996;36:S46.

[11] Bausset 0, Giraudo L, Veran J, et al. Formulation and storage of platelet-rich plasma homemade product. Bioresearch Open Access 2012;1(3):115-23.

[12] Dhurat R, Sukesh M. Principles and methods of preparation of platelet-rich plasma: a review and author's perspective. J Cutan Aesthet Surg 2014;7(4):189-97.

[13] Akhundov K, Pietramaggiori G, Waselle L, et al. Development of a cost-effective method for platelet-rich plasma (PRP) preparation for topical wound healing. Annals of Burns and Fire Disasters 2012;25(4):207-13.

[14] Fukaya M, Asako I. A new economic method for preparing platelet-rich plasma. Plastic and Reconstructive Surgery Global Open 2014;2(6): e162.

[15] Kakaiya K, Aronson CA, Julleis J. Whole blood collection and component processing. In: Roback JD, Combs MR, Grossman BJ, et al. eds. Technical manual. $16^{\text {th }}$ edn. AABB 2008.

[16] Araki J, Jona $M$, Eto $H$, et al. Optimized preparation method of platelet-concentrated plasma and noncoagulating platelet-derived factor concentrates: maximization of platelet concentration and removal of fibrinogen. Tissue Eng Part C Methods 2012;18(3):17685.

[17] Perez AGM, Lana JFSD, Rodrigues AA, et al. Relevant aspects of centrifugation step in the preparation of platelet-rich plasma. Article ID 176060, ISRN Hematology 2014;2014: p.8. http://dx.doi.org/$10.1155 / 2014 / 176060$ 